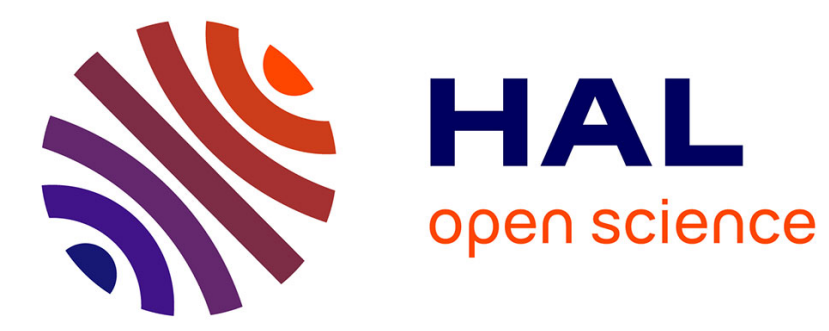

\title{
Cognitive impairments in the STOP null mouse model of schizophrenia.
}

\author{
Kelly J. Powell, Sarah E. Hori, Ronald Leslie, Annie Andrieux, Heather \\ Schellinck, Michael S. Thorne, George S. Robertson
}

\section{To cite this version:}

Kelly J. Powell, Sarah E. Hori, Ronald Leslie, Annie Andrieux, Heather Schellinck, et al.. Cognitive impairments in the STOP null mouse model of schizophrenia.. Behavioral Neuroscience, 2007, 121 (5), pp.826-35. 10.1037/0735-7044.121.5.826 . inserm-00380034

\section{HAL Id: inserm-00380034 https://www.hal.inserm.fr/inserm-00380034}

Submitted on 13 May 2009

HAL is a multi-disciplinary open access archive for the deposit and dissemination of scientific research documents, whether they are published or not. The documents may come from teaching and research institutions in France or abroad, or from public or private research centers.
L'archive ouverte pluridisciplinaire HAL, est destinée au dépôt et à la diffusion de documents scientifiques de niveau recherche, publiés ou non, émanant des établissements d'enseignement et de recherche français ou étrangers, des laboratoires publics ou privés. 


\section{Cognitive Impairments in the STOP Null Mouse Model of Schizophrenia}

Abbreviated Title: Cognition in STOP Null Mice

Kelly J. Powell ${ }^{2}$, Sarah E. Hori ${ }^{2}$, Ronald Leslie ${ }^{3}$, Annie Andrieux ${ }^{5}$, Heather Schellinck ${ }^{4}$, Michael Thorne ${ }^{2}$ and George S. Robertson ${ }^{1,2^{*}}$

Departments of Psychiatry ${ }^{l}$ and Pharmacology ${ }^{2}$, Department of Anatomy and Neurobiology, Sir Charles Tupper Medical Building Faculty of Medicine, Dalhousie University, 5850 College Street, Halifax, Nova Scotia, B3H 1X5, Canada

Department of Psychology, Dalhousie University, Halifax, Nova Scotia, B3H 4J1, Canada

Laboratoire du Cytosquelette ${ }^{5}$

INSERM U366, Département Réponse et Dynamique Cellulaire, CEAGrenoble, 17 Rue des Martyrs, 38054 Grenoble, France;

* Author to whom correspondence should be addressed:

George S. Robertson, PhD

Departments of Psychiatry and Pharmacology,

Sir Charles Tupper Medical Building

Faculty of Medicine, Dalhousie University,

5850 College Street,

Halifax, Nova Scotia, B3H 1X5,

Canada

Phone: 902-494-1528

FAX: 902-494-1388

E-mail: George.Robertson@dal.ca 
Abstract

Background: Cognitive dysfunction is a primary and persisting core deficit of schizophrenia that is marginally improved by antipsychotic treatment. Adult mice that lack the stable tubule-only polypeptide (STOP) have neurochemical and behavioural abnormalities that model some features of schizophrenia.

Methods: Recognition and long-term memory in the STOP null mouse were tested using the novel object recognition task and an olfactory discrimination task, respectively. The brains from STOP null mice were examined to determine whether differences in task performance were associated with alterations in brain morphology

Results: STOP null mice display deficits in both recognition and long-term memory. STOP null mice display massive enlargement of the cerebral ventricular system, accompanied by reductions in volume of cortical and diencephalic structures.

Conclusions: STOP null mice display recognition, long-term memory and neuroanatomical deficits reminiscent of those observed in schizophrenia.

Keywords: Schizophrenia, cognition, ventricular enlargement, microtubule associated protein 
Cognitive deficits associated with schizophrenia have become increasingly recognized as central to both the diagnosis and treatment of this psychiatric disorder (Elvevag and Goldberg 2000). These deficits include problems with attention and perception, problem solving, short- and long-term memory and, in particular, working memory, that often occur prior to the first psychotic episode and persist throughout the course of illness (Peuskens et al 2005). Unfortunately, these cognitive impairments are largely unresponsive to current antipsychotic treatments (Hagan and Jones 2005).

Currently there is no universal animal model with which to study schizophrenia. Existing models are based on both the neurodevelopmental and genetic principles thought to underlie the disorder. Neurodevelopmental models are based on the hypothesis that an intrauterine or early life event results in abnormal brain organization and maturation that leads to the emergence of psychosis in late adolescence or early adulthood (Rehn and Rees 2005). These models involve the experimental manipulation of environmental factors in the pre- or perinatal period of animals and generally result in a schizophrenic phenotype that develops at adulthood (Boksa 2004; Lipska et al 1993; Ozawa et al 2006; Zuckerman et al 2003).

The genetics of schizophrenia are quite complex, and research indicates a relationship between various genetic polymorphisms and their interactions with the chemical environment during development. There are several chromosomal regions encoding putative schizophrenia susceptibility genes (SSGs) that have been identified from genetic linkage studies within specific pedigrees, including Disrupted-inschizophrenia-1 (DISC-1), neuregulin-1 (Nrg-1), dysbindin, catechol-o-methyl transferase (COMT) and metabotropic-glutamate-receptor-3 (mGluR3) (Harrison and 
Weinberger 2005). These genes encode proteins involved in neurodevelopment, synaptic plasticity and signal transduction that may contribute to the synaptic dysfunction observed in schizophrenia (Harrison and Weinberger 2005). Recently, animal models have been developed in which the functionality of SSGs or other genes involved in these same neural processes have been manipulated. For instance, mice in which one allele of the neuregulin-1 gene (regulating neural development: proliferation, migration, survival and differentiation) has been mutated display mild behavioral abnormalities such as hyperlocomotion and deficits in pre-pulse inhibition (PPI) and latent inhibition that recapitulate some clinical features of schizophrenia (Di Segni et al 2005; Gerlai et al 2000; Liu et al 2005; Rimer et al 2005). As well, mice lacking calcineurin expression in the forebrain exhibit deficits in synaptic plasticity and neurite outgrowth, and display impaired episodic learning and memory (delayed matching-to-sample task), hyperlocomotor activity, reduced social interaction and impaired PPI and latent inhibition reminiscent of the psychotic, negative, and cognitive impairments observed in human patients (Gerber et al 2003; Miyakawa et al 2003; Zeng et al 2001).

Mice lacking the microtubule associated protein stable tubule-only polypeptide (STOP) display synaptic deficits, hyper-dopaminergic activity (increased locomotor activity in response to a novel environment or administration of $d$-amphetamine) and behavioural abnormalities (disrupted pre-pulse inhibition, (PPI) and social withdrawal) that are reminiscent of symptoms observed in schizophrenia (Andrieux et al 2006; Andrieux et al 2002; Brun et al 2005; Fradley et al 2005). STOP null mice also show long-term potentiation (LTP) and long-term depression (LTD) deficits in the hippocampus (Andrieux et al 2002) that may contribute to cognitive impairments in these 
animals. Moreover, behavioural abnormalities displayed by these mice such as hyperactivity of the mesolimbic dopamine system and impaired maternal nurturing behaviour are ameliorated by antipsychotic administration (Andrieux et al 2002; Fradley et al 2005). In contrast, PPI or LTP deficits are not reversed by antipsychotic treatments (Andrieux et al 2002; Fradley et al 2005), a profile that closely resembles the clinical outcome achieved by these drugs in schizophrenic patients (Hagan and Jones 2005).

In the present study, STOP null mice were first tested for the presence of a hyperlocomotive phenotype, as compared to wild-type and heterozygous littermates, to establish consistency with previous studies. The main goal of our study was to determine whether STOP null mice have cognitive deficits in object recognition memory and longterm memory as assessed by the novel object recognition task (NORT) and an olfactory discrimination test, respectively. Furthermore, we also tested the STOP null mice in a buried food test as a control measure for motivation towards food rewards and olfactory skills. A histological examination of the brains from STOP null mice was performed to determine whether differences in task performance between STOP null and wild-type littermates were associated with alterations in brain morphology. Specific regions examined included those previously associated with schizophrenia and cognitive deficits including the cerebral cortex, hippocampus, basal ganglia, lateral ventricle, and thalamus, as well as total brain volume.

\section{Methods}

\section{Animals}


All mice used in this study were generated in-house from heterozygous STOP mice breeding pairs (C57B1/6 and 129S1/Smvlj background (50:50)). Litters yielded the expected Mendelian ratios of $25 \%$ wild-type, $50 \%$ heterozygous and $25 \%$ STOP null mice. Adult mice were group-housed in ventilated cages on a $12 \mathrm{hr}$ light/dark cycle with free access to food and water except during the olfactory discrimination task and the buried food task during which the animals were restricted to 2-4 grams of food per day to maintain $85 \%$ body weight. All experiments were conducted according to the guidelines set forth by the Canadian Council of Animal Care and the Dalhousie University Animal Care Committee.

\section{Locomotor Behaviour}

Individual animals were placed in plexiglass boxes $[45 \mathrm{~cm}(1) \times 24 \mathrm{~cm}(\mathrm{w}) \times 20 \mathrm{~cm}(\mathrm{~h})$; size of standard rat cage] and allowed to roam freely. The distance moved and total time spent moving over a 30 min period was recorded using Ethovision Software (Noldus Information Technology).

\section{Novel Object Recognition Task (NORT)}

The NORT was conducted in an opaque plexiglass arena $[35 \mathrm{~cm}(\mathrm{w}) \times 45.5 \mathrm{~cm}(1) \mathrm{x}$ $36 \mathrm{~cm}(\mathrm{~h})]$ using objects that could not be displaced by the animal (full, unopened Pepsi cans and inverted ceramic vases). Adult male and female mice were habituated to the arena in the absence of objects, for 5 min on each of 2 successive days prior to the test day. On the test day, the animals were habituated to the arena for one minute and then removed and placed in a holding cage while two identical objects were placed along the 
length of the arena in two adjacent corners $(7.5 \mathrm{~cm}$ from each wall). During the Sample Phase, each mouse was placed in the centre of the arena and the time spent exploring each object was recorded over a $5 \mathrm{~min}$ period using stopwatches.

Following a delay of $5 \mathrm{~min}$, during which time the animal was again placed in a holding cage, the animal was returned to the arena for the Choice Phase, with one object from the Sample Phase (familiar object) and a novel object. Individuals unaware of the treatment conditions or mouse genotype recorded the time spent exploring each object over a $3 \mathrm{~min}$ period using stopwatches. Exploration was defined as directing the nose towards the object at a distance less than $1 \mathrm{~cm}$, and included placing one or more paws on the object. Sitting close to or on top of the object was not considered exploration. Between the Sample and Choice Phases, and between subjects, the arena and objects were cleaned with a $10 \%$ ethanol solution to remove odour cues.

The total time spent investigating both objects in the Sample Phase was recorded and compared between the 3 different mouse genotypes. In the Choice Phase the proportion of time spent with the novel object was determined by subtracting the time spent with the familiar object from that spent with the novel object, and dividing this time by the total time spent exploring both objects. The resultant value was a Discrimination Ratio, whereby a value of 0 indicated equal time was spent investigating both objects and a value of 1 indicated that all time was spent investigating the novel object.

\section{Olfactory Digging Task}

The olfactory discrimination task consisted of a four-day training session in which the mice were exposed sequentially to one odour paired with sugar (rewarded conditioned 
stimulus; $\mathrm{CS}+$ ) and a second odour presented in the absence of sugar (unrewarded conditioned stimulus; CS-). During the training phase, mice from the 3 groups were randomly divided such that half received rose odour as $\mathrm{CS}+$ and lemon odour as the CSwhile the other half received the opposite reinforcement contingence: lemon $(\mathrm{CS}+) /$ rose odour (CS-). Each odour was presented on filter paper (Whatman no. 1, $55 \mathrm{~mm}$ diameter) that was placed on the bottom of a plastic beverage cup, cut to a $1.5 \mathrm{~cm}$ height and covered with a plastic Petri dish cover containing 10 pre-drilled holes (odour pot). Mice were exposed to the odour stimuli in $30 \mathrm{~cm} \times 13 \mathrm{~cm} \times 11.5 \mathrm{~cm}$ polycarbonate opaque cages, similar to their home cages, with the odour pots buried in Prochip $\mathbb{}$ bedding (PWI Industries, St. Hyacinthe, Que., Canada).

All animals received four 5-min training trials per day (two rose trials and two lemon trials, presented in a quasi-random order), with a 10-min interval between each trial. If an odour was paired with sugar reinforcement, the sugar $(0.05 \mathrm{~g})$ was buried in the bedding covering the odour pots. During each 10-min inter-trial interval, the bedding and filter paper in the test cages were changed and fresh odours applied to the filter paper.

To determine whether the mice had learned the association between an odour and sugar reward, odour discrimination tests were performed $24 \mathrm{hrs}$ and 7 days following the last training day in a $69 \mathrm{~cm} \times 20 \mathrm{~cm} \times 20 \mathrm{~cm}$ box with two pieces of acrylic dividing the apparatus into 3 equal chambers. Prochip $®$ bedding was distributed over the floor of the box. Prior to testing, the animals were habituated for 2 min to the apparatus and then returned to their home cages. For the testing phase, a rose odour pot was placed at one end of the apparatus in one chamber and a lemon odour pot at the other end in a separate 
chamber. The odour pots contained Prochip ${ }^{\circledR}$ bedding but no sugar. The time the mouse spent digging in each odour pot was recorded over a 3-min period. Digging was defined as moving of the bedding with either the forepaws or nose. The apparatus was cleaned and new odour pots and Prochip $®$ bedding used for each mouse. Data are expressed as net digging time in the $\mathrm{CS}+$ versus the $\mathrm{CS}$-odour pot.

\section{Buried Food Test}

The buried food test was carried out according to previously described procedures with only minor modifications (Dawson et al 2005; Harding and Wright 1979). Testing was conducted over 3 days with habituation on day 1 followed by an experimental session on days 2 and 3 . On day 1 , the mice were given a single trial in which a piece of Frootloop ${ }^{\circledR}$ was placed on top of the Prochip $®$ bedding in the centre of the cage. Each mouse was placed individually into the cage and allowed to explore for $5 \mathrm{~min}$. On return to the home cage, the mice were moderately food deprived overnight. On day 2 , the experimental session consisted of four trials separated by a $10 \mathrm{~min}$ inter-trial period in the home cage. At the beginning of each trial, a piece of Frootloop ${ }^{\circledR}$ was buried beneath $1 \mathrm{~cm}$ of Prochip $®$ bedding in a randomly selected location. The mouse was placed in the cage and the time taken to find the buried Frootloop ${ }^{\circledR}$ was recorded. The mouse was then returned to the home cage for the 10 min inter-trial period. If the Frootloop ${ }^{\circledR}$ was not found within 5 min, then 300s was recorded for that trial. Each mouse received four trials; bedding was changed and a new Frootloop ${ }^{\circledR}$ used for each trial. On day 3, another four trial experimental session was completed in an identical manner to the session on day 2 . 
Measurements of brain volume and volumes of cerebral cortex, corpus striatum, thalamus, hippocampus and lateral ventricles

Animals received an overdose of sodium pentobarbital and were perfused intracardially with saline followed by $4 \%$ paraformaldehyde. Brains were removed, post-fixed in $4 \%$ paraformaldehyde and cryoprotected in sucrose prior to sectioning $(30 \mu \mathrm{m})$ on a freezing microtome. The brain was sectioned in the coronal plane in an anterior-to-posterior direction, extending from $1.1 \mathrm{~mm}$ anterior to bregma to $3.28 \mathrm{~mm}$ posterior to bregma (see Paxinos and Franklin, 2004). Brain sections were mounted on Superfrost slides, stained for Niss1 substance with cresyl violet, dehydrated and coverslipped. Images of whole brain sections were visualized using a Zeiss (Axioscope) microscope fitted with a $1 \mathrm{x}$ objective lens. Images were captured with an Axiocam HRc digital camera using imaging software (AxioVision, Zeiss). ScionImage software (Scion Corporation) was used to measure areas in individual sections of the entire brain, thalamus, lateral ventricles, corpus striatum (including the nucleus accumbens shell and core in the measurements), cerebral cortex, and hippocampus (results for bilateral structures were summed over the right and left sides from each animal). Area measurements were computed in square millimeters. Principles of unbiased stereology, according to the Cavalieri method, were used to determine volumes of the measured structures (Mouton 2002). The formula used for the area-to-volume conversions was: Vref $=\left(\Sigma A_{1 \rightarrow i}\right) T$, where Vref refers to the calculated volume, $\mathrm{A}$ is the area on the face of each section 1 to $i, \Sigma A_{1 \rightarrow i}$ is the sum of reference areas in sections 1 to $i$, and $T$ is the distance between each section. We measured every twelfth section (the first measured section being determined randomly), and sections were cut at $30 \mu \mathrm{m}$ thickness, hence $\mathrm{T}=360 \mu \mathrm{m}$ 
$(0.36 \mathrm{~mm})$ in our calculations. For each of the 3 genotypes studied (wild-type, heterozygous and homozygous STOP null mice), 6-9 animals were examined per group; the resultant data are presented as mean values \pm SEM of the volumes, expressed in cubic millimeters $\left(\mathrm{mm}^{3}\right)$.

Statistical Analysis

All data are expressed as the mean \pm S.E.M. A one-way analysis of variance (ANOVA) followed by Newman-Keuls post-hoc tests, where required, was used to assess statistical differences between genotypes in locomotor behaviour, the NORT, and time spent digging in the olfactory discrimination test. A chi-square test was used to compare the number of diggers and non-diggers in the olfactory discrimination task. In the buried food task, statistical differences were determined using a repeated measures two-way ANOVA followed by Bonferroni post-hoc testing, with genotype and trial number as independent variables. A one-way ANOVA followed by Newman-Keuls post-hoc testing was used to detect differences in the volumes of whole brain, thalamus, ventricle, striatum, cortex and hippocampus. All statistical tests were performed using GraphPad Prism version 4.00 for Windows (GraphPad Software, San Diego California, USA).

\section{Results}

In previous studies, it was shown that STOP null mice display a hyperlocomotive phenotype, suggestive of hyperactivity of the mesolimbic dopamine system. Comparison of the total distance moved over a 30 min period, revealed that STOP mull mice moved a significantly longer distance than did wild-type and heterozygous mice (Figure 1A; ANOVA, $\mathrm{F}=4.117, \mathrm{p}=0.03$ ). In addition, the STOP null mice spent significantly more 
time moving over the $30 \mathrm{~min}$ period (Figure $1 \mathrm{~B}$; ANOVA, $\mathrm{F}=4.425, \mathrm{p}=0.02$ ), indicating a basal level of locomotor activity that is greater in the STOP null versus wild-type and heterozygous mice.

The NORT exploits a rodent's innate preference for novelty and is a measure of object recognition memory. During the Sample Phase of the NORT (Figure 2A), no significant differences were found in the amount of time that the wild-type, heterozygous and STOP null mice spent exploring the two identical objects (ANOVA, $F=1.588$, $\mathrm{p}=0.22$ ), suggesting that all mice were motivated to perform this task. During the choice phase(Figure 2B); however, when a familiar object from the sample phase was replaced with a novel object, a comparison of Discrimination Ratios revealed that STOP null mice spent significantly less time exploring the novel object compared to wild-type and heterozygous STOP mice (ANOVA, $\mathrm{F}=5.045, \mathrm{p}=0.01$ ). Thus, STOP null mice were less able to recognize or distinguish a familiar object following a delay of $5 \mathrm{~min}$, suggesting a profound deficit in novel object recognition memory. There was no significant difference between the performance of the wild-type and heterozygous STOP mice.

Long-term memory was assessed using an olfactory discrimination task that required the animals to learn and remember an odour-reward based association. During the training phase, all animals approached both the CS+ and CS- odour pots and were observed digging for, and eating the buried sugar upon presentation in the $\mathrm{CS}+$ odour pot. Twenty-four hours following the four-day training period, all three groups of mice showed a distinct preference for the odour that was previously associated with a reward as demonstrated by digging behaviour predominantly in the CS+ odour pot that lasted for approximately $21 \mathrm{~s}$ (Figure $3 \mathrm{~A}$ ). In the rare instance that a mouse was observed digging in 
the CS- odour pot, it was for a period of only 1-2 seconds, and all graphed data reflects the net time digging in the CS+ odour pot. Although the STOP null mice spent an average amount of time digging that was not significantly different from wild-type or heterozygous STOP mice (ANOVA, $\mathrm{F}=0.1064, \mathrm{p}=0.90$ ), several STOP null animals did not display digging behaviour in the previously rewarded odour pot (Figure 3A). In fact, 6 of the 17 STOP null mice tested did not dig at all, compared to only 2 heterozygous mice and one wild-type mouse. A chi-square analysis revealed a significant difference in the number of diggers versus non-diggers in the groups of wild-type, heterozygous and STOP null mice (Figure $2 \mathrm{~B}$, chi-square $=6.039, \mathrm{df}=2, \mathrm{p}=0.048$ ). These results suggest that a significant number of the STOP null mice were unable to either learn the odour-reward based association or remember this association $24 \mathrm{hrs}$ post-training.

Memory for the odour-reward based association was also tested at 7 days following training. As shown in Figure 3C, the wild-type and heterozygous STOP mice still showed a preference for the previously rewarded odour indicated by elevated time spent digging in the odour pot associated with reward. In contrast, STOP null mice spent less time digging in the previously rewarded odour pot compared to wild-type and heterozygous mice (ANOVA, $\mathrm{F}=3.311, \mathrm{p}=0.046$ ). Interestingly, most of the STOP null mice did not dig for the reward at all, compared to approximately one-half of the heterozygous mice and only 2 of the wild-type mice. A chi-square analysis revealed a significant difference in the number of diggers versus non-diggers in the wild-type, heterozygous and STOP null mice at this 7 day time point (Figure 3D; chi-square $=13.45$, $\mathrm{df}=2, \mathrm{p}=0.001$ ). These results indicate that STOP null mice (are unable) (have greater 
difficulty) to remember an odour-reward based association following a 7 day delay after training, suggesting that these mice may have deficits in long-term memory.

The olfactory discrimination task relies on the fact that food-deprived animals are motivated to dig for food reward, in this case sugar, and will associate the reward with an odour. The deficits displayed by the STOP null mice in the olfactory digging task would be confounded if the animals were not motivated to search for a food reward or suffered from olfactory deficits. To assess olfaction in the STOP null mice we examined their performance in a buried food test. Following food deprivation, the wild-type and STOP null mice actively searched for, found and ate the buried Frootloop ${ }^{\circledR}$. On average, mice were able to find the buried Frootloop ${ }^{\circledR}$ in under the 300 second cut-off on the first day of testing and the time to perform this task further decreased over successive trials on the second day (Figure 4). Consequently, wild-type and STOP null mice performed at comparable levels in this test, with no significant effect of genotype or trial session on either day. These data suggest that STOP null mice can smell and are motivated to find a food reward, implying that memory deficits rather than olfactory or motivational impairments were responsible for deficits in the olfactory digging test.

Following completion of the behavioural tasks, all mice were sacrificed and brain sections analyzed for the presence of morphological differences between groups. Representative photomicrographs of brain sections taken from several brain levels are shown in Figures 5 and 6. These figures clearly demonstrate that STOP null mice display a profound enlargement of the lateral ventricles accompanied by smaller volumes of cerebral cortical and subcortical regions compared to wild-type mice. Heterozygous STOP mice exhibited similar, but less obvious abnormalities that were determined to be 
significant in quantitative experiments (see Figure 7). Quantitative measurements indicated that STOP null mice display significantly larger cerebral ventricular volumes compared to wild-type $(\mathrm{p}<0.05)$, but not heterozygous, mice (ANOVA, $F=5.108$, $p=0.0175$; Figure $7 \mathrm{~A}$ ). Although there was an overall trend towards a smaller total brain size in STOP null mice compared to heterozygous and wild-type mice, there was no significant difference in total brain volume between the three different genotypes (ANOVA, $\mathrm{F}=1.852, \mathrm{p}=0.1829 ;$ Figure 7B). However, the STOP null mice had significantly smaller striatal volumes (ANOVA, $\mathrm{F}=6.123, \mathrm{p}<0.0084$ ) in comparison with wild-type mice $(p<0.01)$, but not heterozygotes (Figure 7C). Measurements of the thalamic nuclei (ANOVA, F=18.72, $\mathrm{p}<0.001$ ) revealed that STOP null mice had significantly smaller volumes compared to wild-type $(\mathrm{p}<0.001)$ and STOP $(+/-)$ mice $(p<0.001)$ (Figure 7D). STOP null mice also displayed significantly smaller cerebral cortical volumes (ANOVA, $F=5.417, p=0.0138)$ in comparison with wild-type $(\mathrm{p}<0.05)$ and heterozygous STOP mice $(p<0.05$; Figure 7E). In contrast, measurements of hippocampal volumes revealed no significant differences between wild-type, heterozygous and STOP null mice (ANOVA, F0.5596, p=0.5596; Figure 7F).

\section{Discussion}

The major finding of the present manuscript is that STOP null mice display cognitive deficits in recognition memory. We also found that lack of the STOP protein leads to developmental abnormalities in brain structure resulting in an enlarged ventricular system accompanied by smaller than normal cerebral cortical, striatal and thalamic volumes. These changes may contribute to the performance deficits in tasks requiring object recognition or long-term memory. 
Cognitive deficits in schizophrenia are thought to result from synaptic loss or aberrant connectivity (Glantz et al 2006; Lewis and Lieberman 2000). Microtubule associated proteins, such as STOP, play an integral role in the formation and maintenance of synaptic connections, vesicle trafficking and cell migration (Bosc et al 2003). The importance of these types of microtubule associated proteins is demonstrated by the fact that deletion of doublecortin leads to massive alterations in cortical development and results in a lissencephaly characterized by mental retardation (Francis et al 1999). Deletion of the STOP protein appears to result in a more subtle phenotype, as it is difficult to distinguish STOP null mice from wild-type and heterozygous littermates upon initial observation. Nevertheless, it has been demonstrated that the STOP protein plays an important role in microtubule stability and is responsible for stability under conditions of cold- and drug- (nocodazole) induced stress in vitro (Bosc et al 1996). Furthermore, inhibition of STOP activity in vitro leads to neuronal microtubule disassembly and hindered neurite formation, suggesting STOP plays an important role in the normal structure and function of neurons (Guillaud et al 1998). STOP isoforms, all of which contain exon 1 of the stop gene, are present in astrocytes and oligodendrocytes as well as neurons (Bosc et al 2003). The STOP null mouse was generated by targeted deletion of exon 1 , and thus a lack of STOP in any of these 3 cell types may result in the observed phenotype and cognitive dysfunction in these mice (Andrieux et al 2002).

CT and MRI studies have consistently reported ventricular enlargement in human schizophrenia patients (Shenton et al 2001). Our finding that STOP null mice show a massive enlargement of the cerebral ventricular system is particularly important in terms of further establishing these mice as a suitable model for schizophrenia. Although MRI 
studies of human brain regions have often yielded conflicting results, several studies have shown that patients with schizophrenia display regional decreases in gray matter in the caudate nucleus, putamen and thalamus (Antonova et al 2004; Shenton et al 2001). Given the role of the basal ganglia in dopamine signaling, the importance of these structures as a target for neuroleptic action, and the integral function played by the basal ganglia play in sensory, cognitive and motor processing, it is not surprising that many studies have identified structural anomalies in these brain regions in schizophrenic patients (Shenton et al 2001). Interestingly, the size of the basal ganglia has been correlated with the performance of schizophrenia patients on the Wisconsin Card Sorting Test, with poor performers displaying decreased basal ganglia size relative to controls and good WCST performers diagnosed with schizophrenia (Stratta et al 1997). Similarly, the thalamus is a major relay station in the brain modulating inputs from cortical and limbic structures, and is involved in attention, information processing and sensory gating: processes that may be impaired in schizophrenia (Shenton et al 2001). Although ventricular enlargement is not a finding that is specific to schizophrenia, the fact that STOP null mice display this feature in addition to cortical, striatal and thalamic atrophy as well as cognitive deficits, hyper mesolimbic dopaminergic activity, social withdrawal, disrupted PPI and responsiveness to chronic neuroleptic administration, suggest that these mice may provide a useful experimental paradigm to study some elements of the etiology and treatment of schizophrenia (Andrieux et al 2002; Brun et al 2005; Fradley et al 2005). While there are many other genetic models of schizophrenia that have been developed in recent years [for review see (O'Tuathaigh $\mathrm{C}$ et al 2006)], none has been investigated as thoroughly as the STOP null mouse or has demonstrated as broad an array of neurochemical, 
neuropathological, cognitive and behavioural abnormalities that closely resemble those observed in schizophrenia.

Despite the fact that the STOP null mouse has only recently been characterized as a model of schizophrenia, these mice clearly recapitulate many of the cardinal features of this psychiatric disorder. STOP null mice show deficits in behavioural correlates for all of the major classes of symptoms characteristic of schizophrenia: positive, negative and cognitive. In the present study we have provided more evidence for this, including deficits in delayed object recognition and in the long-term memory of an odour-reward association. Moreover, we have also demonstrated anatomical abnormalities in the STOP null mouse brain, such as ventricular enlargement and abnormally small cortical and subcortical nuclear structures in the diencephalon. These abnormalities are consistent with those that have been observed in some schizophrenic patients. Taken together, these findings suggest that the STOP null mouse may be a useful animal model of schizophrenia for future studies

Acknowledgements: The authors would like to thank Dr. Rebecca Dias for assistance in establishing the novel object recognition test. The authors gratefully acknowledge Rhian Gunn, Kay Murphy, Daniel Costa and Soulatchana Purnima Narayanan for their assistance with the olfactory tests. KJP was supported by a postdoctoral fellowship from CIHR/INMHA/Estate of Betty Irene West. HMS is supported by an NSERC Discovery Grant. GSR is supported by a CIHR Rx\&D Chair. This work was supported in part by a grant from Amgen Inc. and CIHR operating grant MOP-67017. 


\section{References}

Andrieux A, Salin P, Schweitzer A, et al (2006): Microtubule stabilizer ameliorates synaptic function and behavior in a mouse model for schizophrenia. Biol Psychiatry 60:1224-30.

Andrieux A, Salin PA, Vernet M, et al (2002): The suppression of brain cold-stable microtubules in mice induces synaptic defects associated with neurolepticsensitive behavioral disorders. Genes Dev 16:2350-64.

Antonova E, Sharma T, Morris R, Kumari V (2004): The relationship between brain structure and neurocognition in schizophrenia: a selective review. Schizophr Res 70:117-45.

Boksa P (2004): Animal models of obstetric complications in relation to schizophrenia Brain Res Brain Res Rev 45:1-17.

Bosc C, Andrieux A, Job D (2003): STOP proteins. Biochemistry 42:12125-32.

Bosc C, Cronk JD, Pirollet F, et al (1996): Cloning, expression, and properties of the microtubule-stabilizing protein STOP. Proc Natl Acad Sci US A 93:2125-30.

Brun P, Begou M, Andrieux A, et al (2005): Dopaminergic transmission in STOP null mice. J Neurochem 94:63-73.

Dawson PA, Steane SE, Markovich D (2005): Impaired memory and olfactory performance in NaSi-1 sulphate transporter deficient mice. Behav Brain Res 159:15-20.

Di Segni A, Shaharabani E, Stein R, Pinkas-Kramarski R (2005): Neuregulins rescue PC12-ErbB-4 cells from cell death induced by beta-amyloid peptide: involvement of PI3K and PKC. J Mol Neurosci 26:57-69.

Elvevag B, Goldberg TE (2000): Cognitive impairment in schizophrenia is the core of the disorder. Crit Rev Neurobiol 14:1-21.

Fradley RL, O'Meara G F, Newman RJ, Andrieux A, Job D, Reynolds DS (2005): STOP knockout and NMDA NR1 hypomorphic mice exhibit deficits in sensorimotor gating. Behav Brain Res 163:257-64.

Francis F, Koulakoff A, Boucher D, et al (1999): Doublecortin is a developmentally regulated, microtubule-associated protein expressed in migrating and differentiating neurons. Neuron 23:247-56.

Gerber DJ, Hall D, Miyakawa T, et al (2003): Evidence for association of schizophrenia with genetic variation in the $8 \mathrm{p} 21.3$ gene, PPP3CC, encoding the calcineurin gamma subunit. Proc Natl Acad Sci US A 100:8993-8.

Gerlai R, Pisacane P, Erickson S (2000): Heregulin, but not ErbB2 or ErbB3, heterozygous mutant mice exhibit hyperactivity in multiple behavioral tasks. Behav Brain Res 109:219-27.

Glantz LA, Gilmore JH, Lieberman JA, Jarskog LF (2006): Apoptotic mechanisms and the synaptic pathology of schizophrenia. Schizophr Res 81:47-63.

Guillaud L, Bosc C, Fourest-Lieuvin A, et al (1998): STOP proteins are responsible for the high degree of microtubule stabilization observed in neuronal cells. J Cell Biol 142:167-79.

Hagan JJ, Jones DN (2005): Predicting drug efficacy for cognitive deficits in schizophrenia. Schizophr Bull 31:830-53. 
Harding JW, Wright JW (1979): Reversible effects of olfactory nerve section on behavior and biochemistry in mice. Brain Res Bull 4:17-22.

Harrison PJ, Weinberger DR (2005): Schizophrenia genes, gene expression, and neuropathology: on the matter of their convergence. Mol Psychiatry 10:804.

Lewis DA, Lieberman JA (2000): Catching up on schizophrenia: natural history and neurobiology. Neuron 28:325-34.

Lipska BK, Jaskiw GE, Weinberger DR (1993): Postpubertal emergence of hyperresponsiveness to stress and to amphetamine after neonatal excitotoxic hippocampal damage: a potential animal model of schizophrenia. Neuropsychopharmacology 9:67-75.

Liu Y, Ford BD, Mann MA, Fischbach GD (2005): Neuregulin-1 increases the proliferation of neuronal progenitors from embryonic neural stem cells. Dev Biol 283:437-45.

Miyakawa T, Leiter LM, Gerber DJ, et al (2003): Conditional calcineurin knockout mice exhibit multiple abnormal behaviors related to schizophrenia. Proc Natl Acad Sci $U S A$ 100:8987-92.

Mouton P (2002): Principles and Practices of Unbiased Stereology. The Johns Hopkins University Press:Baltimore, MD, USA.

O'Tuathaigh C M, Babovic D, O'Meara G, Clifford JJ, Croke DT, Waddington JL (2006): Susceptibility genes for schizophrenia: Characterisation of mutant mouse models at the level of phenotypic behaviour. Neurosci Biobehav Rev.

Ozawa K, Hashimoto K, Kishimoto T, Shimizu E, Ishikura H, Iyo M (2006): Immune activation during pregnancy in mice leads to dopaminergic hyperfunction and cognitive impairment in the offspring: a neurodevelopmental animal model of schizophrenia. Biol Psychiatry 59:546-54.

Peuskens J, Demily C, Thibaut F (2005): Treatment of cognitive dysfunction in schizophrenia. Clin Ther 27 Suppl A:S25-37.

Rehn AE, Rees SM (2005): Investigating the neurodevelopmental hypothesis of schizophrenia. Clin Exp Pharmacol Physiol 32:687-96.

Rimer M, Barrett DW, Maldonado MA, Vock VM, Gonzalez-Lima F (2005):

Neuregulin-1 immunoglobulin-like domain mutant mice: clozapine sensitivity and impaired latent inhibition. Neuroreport 16:271-5.

Shenton ME, Dickey CC, Frumin M, McCarley RW (2001): A review of MRI findings in schizophrenia. Schizophr Res 49:1-52.

Stratta P, Mancini F, Mattei P, Daneluzzo E, Casacchia M, Rossi A (1997): Association between striatal reduction and poor Wisconsin card sorting test performance in patients with schizophrenia. Biol Psychiatry 42:816-20.

Zeng H, Chattarji S, Barbarosie M, et al (2001): Forebrain-specific calcineurin knockout selectively impairs bidirectional synaptic plasticity and working/episodic-like memory. Cell 107:617-29.

Zuckerman L, Rehavi M, Nachman R, Weiner I (2003): Immune activation during pregnancy in rats leads to a postpubertal emergence of disrupted latent inhibition, dopaminergic hyperfunction, and altered limbic morphology in the offspring: a novel neurodevelopmental model of schizophrenia. Neuropsychopharmacology 28:1778-89. 


\section{Figure Legends}

Figure 1. Locomotor behaviour of wild-type, heterozygous and STOP null mice in an open field, measured as both distance moved (A) and time spent moving (B). *Significantly different from wild-type and heterozygous in both distance moved $(p=0.03)$ and time spent moving $(p=0.02)$. Data are presented as mean \pm S.E.M. $n=7-9$ /genotype

Figure 2. Performance of wild-type, heterozygous and STOP null mice in the sample phase (A) and choice phase (B) of the novel object recognition task. ${ }^{*}$ Significantly different from wild-type $(p<0.01)$ and heterozygous mice $(p<0.05)$. Data are presented as mean \pm S.E.M. $n=12-15 /$ genotype.

Figure 3. Performance of wild-type, heterozygous and STOP null mice in the olfactory digging task $24 \mathrm{hrs}(\mathrm{A}, \mathrm{B})$ and 7 days $(\mathrm{C}, \mathrm{D})$ post-training. Average time spent digging by wild-type, heterozygous and STOP null mice in the previously rewarded odour pot at 24 hrs (A) and 7 days (C) post training $\left({ }^{*} p<0.05, n=15-19 /\right.$ genotype). Profile of the number of wild-type, heterozygous and STOP null mice that spent time digging or spent no time digging in the rewarded odour pot at $24 \mathrm{hrs}(\mathrm{B})$ and 7 days (D) post training.

Figure 4. Performance of wild-type and STOP null mice in the buried food test over four trials conducted on 2 successive days. Data are presented as mean \pm S.E.M. $n=7 /$ genotype. 
Figure 5. Photomicrographs of cresyl violet stained coronal brain sections from wildtype (WT; A,B,C) and STOP null mice (STOP null; D,E,F) at brain levels extending from 1.10 to $-0.70 \mathrm{~mm}$ from bregma. Enlargement of the ventricles as well as abnormally small striatal regions are evident in sections from STOP null mice.

Figure 6. Photomicrographs of cresyl violet stained coronal brain sections from wildtype (WT; A,B,C) and STOP null mice (STOP null; D,E,F) at brain levels extending from -1.06 to $-2.92 \mathrm{~mm}$ from bregma. Enlargement of the ventricles as well as abnormally small thalamic nuclei are evident in sections from STOP null mice. By contrast, ventral hippocampal morphology was similar in STOP null and WT littermates. Although dorsal hippocampal morphology appeared different in STOP null and WT littermates, there were no volumetric statistical differences in this structure

Figure 7. Volume $\left(\mathrm{mm}^{3}\right)$ comparisons of the lateral ventricles (A), entire brain (B), corpus striatum (C), thalamus (D), cerebral cortex (E) and hippocampus $(F)$ in wild-type, heterozygous and STOP null mice. Volumes of the lateral ventricles and corpora striata are significantly smaller in STOP null mice compared to wild-type animals $\left(^{*}\right)$. Volumes of thalamic nuclei and cerebral cortex are significantly smaller than those of both wildtype and heterozygous mice $(*)$. Data are presented as mean \pm S.E.M. 